ARTICLE

\title{
Arabidopsis calcium-dependent protein kinase 3 regulates actin cytoskeleton organization and immunity
}



Pattern-triggered immunity and effector-triggered immunity are two primary forms of innate immunity in land plants. The molecular components and connecting nodes of patterntriggered immunity and effector-triggered immunity are not fully understood. Here, we report that the Arabidopsis calcium-dependent protein kinase CPK3 is a key regulator of both pattern-triggered immunity and effector-triggered immunity. In vitro and in vivo phosphorylation assays, coupled with genetic and cell biology-based analyses, show that actindepolymerization factor 4 (ADF4) is a physiological substrate of CPK3, and that phosphorylation of ADF4 by CPK3 governs actin cytoskeletal organization associated with patterntriggered immunity. CPK3 regulates stomatal closure induced by flg22 and is required for resistance to Pst DC3000. Our data further demonstrates that CPK3 is required for resistance to Pst DC3000 carrying the effector AvrPphB. These results suggest that CPK3 is a missing link between cytoskeleton organization, pattern-triggered immunity and effectortriggered immunity.

\footnotetext{
${ }^{1}$ Department of Plant, Soil and Microbial Sciences, Michigan State University, East Lansing, MI 48824, USA. ${ }^{2}$ Department of Plant Biology, Michigan State University, East Lansing, MI 48824, USA. ${ }^{3}$ International Research Organization for Advanced Science and Technology (IROAST), Kumamoto University, Kumamoto 860-8555, Japan. ${ }^{4}$ Department of Energy Plant Research Laboratory, East Lansing, MI 48824, USA. ${ }^{5}$ Howard Hughes Medical Institute, Michigan State University, East Lansing, MI 48824, USA. ${ }^{凶}$ email: bday@msu.edu
} 


\section{T} he actin-depolymerizing factor (ADF) and cofilin family of proteins function as key regulators of actin cytoskeletal dynamics ${ }^{1,2}$, responsible for controlling numerous aspects of cytoskeletal organization, including filament growth, stability, organization, and disassembly. Additionally, ADF activity influences the size and distribution of the actin monomer pool, a process that controls the rapid assembly, growth, and orientation of the cytoskeletal network within a cell. Underpinning each of these processes is phosphorylation, a key step controlling ADFactin association and depolymerization activity ${ }^{3}$. In mammalian systems, the cofilin phospho-switch is regulated by LIM (LIMK1 and LIMK2) and TES (TESK1 and TESK2) protein kinases ${ }^{4,5}$. In plants, this process is proposed to be functionally similar; however, despite the identification of several ADF-phosphorylating kinases $^{6-8}$, the kinase responsible for ADF phosphorylation relevant to plant-pathogen interactions remains enigmatic.

Recent studies demonstrate a role for the plant actin cytoskeleton in pathogen recognition and the activation of both PTI and $\mathrm{ETI}^{9-17}$. As a function of ETI, ADF4 has been shown to be required for immune signaling mediated by the nucleotidebinding leucine-rich repeat resistance (R) protein RPS5, a process that is initiated following recognition of the type-III effector (T3E) AvrPphB, a cysteine protease ${ }^{13,16}$. A key step in the activation of RPS5 is AvrPphB cleavage of the receptor-like cytoplasmic kinase PBS1 (AvrPphB Susceptible-1) by AvrPphB ${ }^{18,19}$, a mechanism hypothesized to be associated with R-protein destabilization and immune activation. In addition to R-protein activation, recent work has identified $\sim 10$ PBS1-like kinases that are cleaved by $\mathrm{AvrPphB}^{20}$, among which includes the immune kinase BIK1 (Botrytis-induced kinase-1) ${ }^{21}$. This is significant, as it implicates BIK $\overline{1}$ and additional immune kinases as potential regulators of distinct pathogen recognition events in plants, integrating immune responses activated through multiple pattern recognition receptors, including FLS2 (Arabidopsis flagellin receptor $^{22}$ ), EFR (receptor for bacterial Ef-Tu' ${ }^{23}$ ), and CERK1 (chitin receptor ${ }^{23}$ ). As a broader function of this signaling cascade, recent work has shown that the actin cytoskeleton is not only required for the activity of the plant immune system, but that actin is actively targeted by pathogens during infection ${ }^{15,24}$. In the case of PTI, it has been demonstrated that pathogenassociated molecular pattern (PAMP) recognition requires the function of the plant actin cytoskeleton, presumably to initiate downstream signaling associated with resistance ${ }^{10}$. However, the mechanistic connection between the requirement of ADF4 in RPS5-mediated ETI, AvrPphB-mediated cleavage of immune kinases, and PTI remains a puzzle.

In the current work, we present the identification of a kinase responsible for ADF4 phosphorylation and subsequent control of actin filament association and depolymerization during immune signaling. Using a combination of in vitro and in vivo kinase assays coupled with a mass spectroscopy-based approach, we show that the Arabidopsis calcium-dependent protein kinase, $\mathrm{CPK} 3$, is responsible for ADF4 phosphorylation, a process that regulates actin cytoskeletal dynamics. We also demonstrate that $\mathrm{CPK} 3$ is required for proper stomatal actin filament organization and PTI-induced guard cell gating. In addition, analysis of the immune signaling function of the $c p k 3$ 2 mutant revealed that inoculation of plants with Pst DC3000 expressing AvrPphB (Pst-AvrPphB) phenocopies the adf4 mutant ${ }^{13,16}$, with significant increases in the in planta growth of the pathogen and an absence of the hypersensitive response (HR). In total, these data support a model whereby CPK3 plays an important role in PTI and, through its phosphorylation of ADF4 is also required for ETI activation by pathogen effectors (e.g., cysteine protease AvrPphB) that destabilize immune receptor-guarded proteins.

\section{Results}

CPK3 phosphorylates the actin-depolymerizing factor ADF4. Previous work identified a genetic interaction between ADF4dependent changes in actin cytoskeletal organization and the activation of the AvrPphB-RPS5 immune signaling node ${ }^{13,16}$. To define the mechanism(s) underpinning changes in actin cytoskeletal organization during pathogen infection, and moreover, the regulation of ADF4, we sought to identify the kinase responsible for ADF4 regulation. Leveraging previously published data showing phosphorylation of $\mathrm{ADF} 1$ by $\mathrm{CPK}^{7}$, we first performed in vitro kinase assays using recombinant CPK3, CPK6, and CPK28 - each of which represent a distinct clade of the CPK family-to interrogate the role of CPK in the regulation of ADF4. Recombinant ADF4 was preferentially phosphorylated by CPK3, while ADF4 was nominally phosphorylated by CPK6 and CPK28 (Fig. 1a, Supplementary Fig. 1, and Supplementary Fig. 2a). As expected, the kinase-inactive variant, $\mathrm{CPK} 3{ }^{\mathrm{K} 107 \mathrm{M}}$, did not autophosphorylate (Supplementary Fig. 2b), nor did it phosphorylate ADF4 (Supplementary Fig. 2c).

To identify CPK3 phosphorylation sites on ADF4, we first employed an in silico-based approach, the result of which revealed high-confidence predictions for phosphorylation of ADF4 at Ser ${ }^{105}$, with moderate phosphorylation at $\operatorname{Ser}^{106}$, as well as minor phosphorylation events at $\operatorname{Ser}^{6}$ (Supplementary Fig. 3a). Mass spectroscopy analysis of in vitro kinase assays confirmed these predictions (Supplementary Fig. 3b, c). As a broader function of the interaction specificity between CPK3 and ADF4, we also tested the in vitro activity of the PTI-associated RLCK BIK1 (Fig. 1b and Supplementary Fig. 1b), as well as the ETI-associated immune RLCK PBS1 (Fig. 1c and Supplementary Fig. 1c) and found that neither kinase phosphorylated ADF4; this result was also confirmed by mass spectroscopy (Supplementary Fig. 4).

To validate the ADF4 phosphorylation residues, we used PCR mutagenesis to convert the predicted high-confidence phosphoserine residues (i.e., $\mathrm{Ser}^{6}$, $\mathrm{Ser}^{105}$, and $\mathrm{Ser}^{106}$ ) to alanine (i.e., S6A, S105A, and S106A), and again performed in vitro kinase assays. Using this approach, we were successful in identifying preferential phosphorylation of ADF4 by CPK3 at $\operatorname{Ser}^{105}$ (Fig. 1d, e and Supplementary Fig. 1d). To validate that phosphorylation of ADF4 by CPK3 occurs in vivo, we performed two-dimensional electrophoresis analysis of Arabidopsis protoplasts prepared from WT Col-0 and the $c p k 3-2$ mutant and transiently expressed the kinase substrate ADF4 containing an HA-epitope tag (i.e., ADF4HA). As shown in Fig. 1f, ADF4-HA expressed in WT Col-0 protoplasts showed a robust phosphorylation. Conversely, and in support of our hypothesis, in protoplasts derived from the $c p k 3-2$ mutant, the kinase-dead CPK3 complementation line (i.e., cpk3$\left.2 / 35 S:: C P K 3^{K 107 M}\right)$, and protoplasts treated with calf intestinal alkaline phosphatase (CIP), ADF4 phosphorylation was significantly reduced. Thus, both in vitro and in vivo phosphorylation assays confirm that ADF4 is a physiological substrate of CPK3.

Next, we asked if Ser ${ }^{105}$ and Ser ${ }^{106}$ are required for ADF4 association with actin in planta. To test this, we performed coimmunoprecipitation assays using WT ADF4, as well as the phospho-null (Ser to Ala) and phosphomimic (Ser to Asp) derivatives, with Arabidopsis actin-7 (ACT7) following transient expression in N. benthamiana. While ADF4 and the phosphornull derivatives $\mathrm{ADF} 4{ }^{\mathrm{S} 105 \mathrm{~A}}$, and $\mathrm{ADF} 44^{\mathrm{S} 106 \mathrm{~A}}$ interacted with $\mathrm{ACT} 7$, the phosphomimic variants $\mathrm{ADF} 4^{\mathrm{S} 105 \mathrm{D}}$, and $\mathrm{ADF} 4^{\mathrm{S} 106 \mathrm{D}}$ did not associate with actin (Fig. 2a and Supplementary Fig. 5). Similar results were observed by transiently expressing WT ADF4 and its phosphomimetic derivatives as red fluorescent protein (RFP) fusions proteins in N. benthamiana in combination with green florescent protein (GFP)-tagged actin-binding domain (fABD2). As shown, the phosphomimic derivatives $\mathrm{ADF} 4^{\mathrm{S} 105 \mathrm{D}}$ 
a

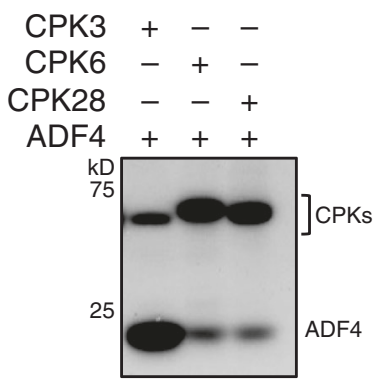

d

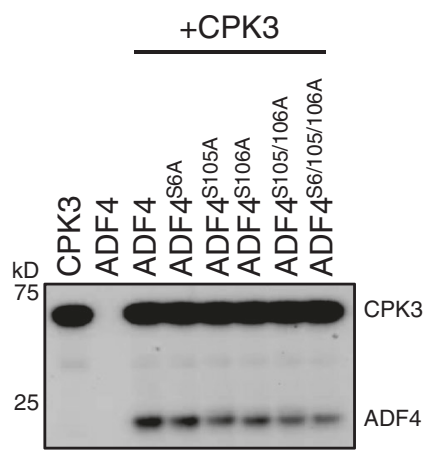

b

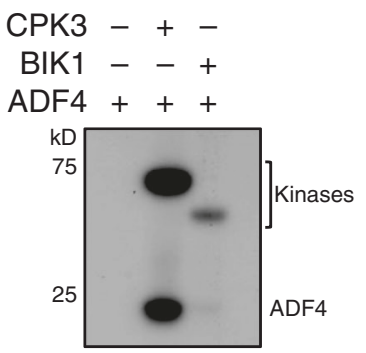

e

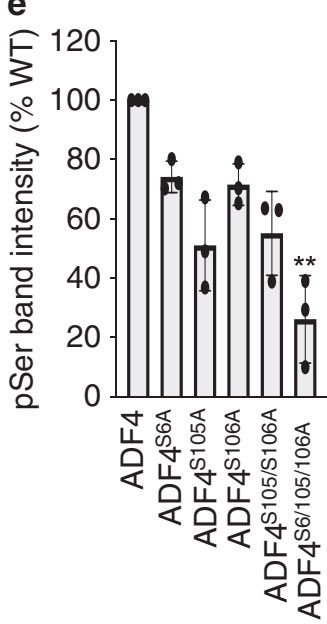

C

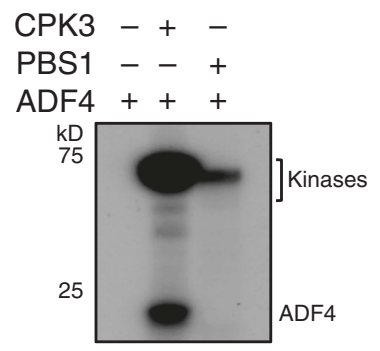

f

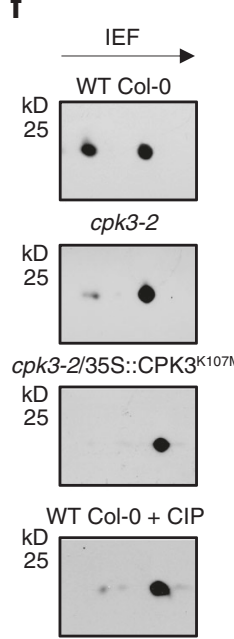

Fig. 1 CPK3 phosphorylates the Arabidopsis actin-depolymerizing factor ADF4. In vitro kinase assays using recombinant CPK3, CPK6, CPK28, and ADF4. a CPK3 predominantly phosphorylates ADF4 as compared to CPK6 and CPK28. The receptor-like cytoplasmic Ser/Thr kinases b BIK1 and c PBS1 do not phosphorylate ADF4. d CPK3 predominantly phosphorylates ADF4 at amino acid residues Ser ${ }^{105}$. a-d Experiments were repeated three times. e Mean \pm SD of densitometry scans from three independent biological replicates of the kinase assays shown in $\mathbf{d}$. Data is represented as a percentage of WT ADF4 phosphorylation. Asterisks denote standard deviation of the mean compared to WT ADF4 (one-way ANOVA, ${ }^{*} p$-value $<0.1 ;{ }^{* \star} p$-value $<0.01$ ). $\mathbf{f}$ Twodimensional gel electrophoresis analysis of HA-tagged ADF4 expressed in WT Col-0, cpk3-2, the kinase inactive-expressing line cpk3-2/CPK3 ${ }^{\mathrm{K} 107 \mathrm{M}}$ and CIP-treated WT Col-0 protoplasts. Arrow indicates the direction of isoelectric focusing (IEF). Experiments were repeated two times.

RFP and ADF4 $106 \mathrm{D}$-RFP did not co-localize with fABD2, while ADF4 ${ }^{\text {S6D }}$-RFPhad attenuated association (Fig. $2 \mathrm{~b}$ and Supplementary Fig. 5). These data support a key role for these three serine residues in F-actin binding by ADF4.

CPK3 is required for proper actin filament organization in epidermal pavement cells and stomatal guard cells during pathogen infection. Having identified $\mathrm{CPK} 3$ as an ADF4 kinase, we next investigated the effect of CPK3 on cytoskeletal organization. Specifically, we examined actin filament architecture in epidermal pavement cells of WT Col-0/35S::GFP-fABD2 and $c p k 3-2 / 35 S:: G F P-f A B D 2$ plants. $c p k 3-2$ mutant plants showed significant decreases in filament occupancy (represented by density) and higher levels of bundling (represented by skewness) in cells from untreated plants. We did not detect a significant difference in the parallel organization of actin filaments (Fig. 3a). Following inoculation of seedlings with virulent Pst DC3000, WT Col-0/GFP-fABD2 plants showed a marked, statistically significant, increase in actin filament bundling (Fig. 3b), consistent with previous observations ${ }^{15}$. Conversely, following inoculation of $c p k 3-2 / 35 S:: G F P-f A B D 2$ with Pst DC3000, we did not observe an increase in actin filament bundling as compared to mockinoculated $c p k 3-2 / 35 S:: G F P-f A B D 2$ (Fig. 3c). These data support a role for $\mathrm{CPK} 3$ in pathogen-induced actin filament (re)-organization. Interestingly, we identified a statistically significant increase in CPK3 mRNA accumulation following flg22 treatment
(Supplementary Fig. 6), indicating a genetic interaction between PTI signaling and CPK3.

Flg22-induced MAPK activation (Supplementary Fig. 7a), the generation of reactive oxygen species (ROS) (Supplementary Fig. 7b) and flg22-triggered priming (Supplementary Fig. 8) were not altered in the cpk3-2 mutant compared to WT Col-0. Unexpectedly, however, we observed reductions in FRK1, PR-1, WRKY70, and WRKY46 mRNAs in the cpk3-2 mutant line following flg22 stimulation (Supplementary Fig. 9). At present, it is unknown if these reductions in mRNAs are due to alternations in actin filament dynamics, or are related to additional, direct or indirect functions of CPK3.

Previous work demonstrated that stomatal guard cell actin filament organization is altered in response to PTI signaling ${ }^{10,14}$. To determine if $\mathrm{CPK} 3$, through its phosphorylation of ADF4, exerts control over the steady-state cellular concentration of Gand F-actin, we assessed the relative abundance of actin in WT Col-0 and the cpk3-2 mutant. As shown in Supplementary Fig. 10 the ratio of total G- and F-actin from 4-week-old plants was similar in both WT Col-0 and the cpk3-2 mutant in both untreated and Pst DC3000-inoculated leaves. Conversely, upon elicitation of flg22-induced PTI, we observed a dramatic shift in the cellular ratios of G- and F-actin; however, we did not detect a significant difference in G- and F-actin ratios between WT Col-0 and the cpk3-2 mutant. Thus, CPK3 does not appear to be required for maintenance of cellular G- and F-actin levels and/or 
$\mathbf{a}$


Fig. 2 Ser-105 and Ser-106 are essential for ADF4 interaction with actin. a Co-immunoprecipitation of ADF4 phosphorylation variants with Arabidopsis actin following transient expression in N. benthamiana; the phosphomimetic variants do not co-immunoprecipitated with Actin. $\mathbf{b}$ In vivo fluorescence microscopy localization of ADF4 variants and actin-binding domain. Constructs were co-expressed in N. benthamiana as N-terminal mRFP fusion proteins for ADF4 variants and a C-terminal GFP fusion protein for fABD2 using A. tumefaciens-mediated transient expression. Arrows indicate co-localization of the fluorescence signal with F-actin. Scale bar $=10 \mu \mathrm{m}$. Experiments were performed three times with similar results, and 30 images were collected in total for each co-expressed construct.

the ratio of the two. Based on this, we surmise that $\mathrm{CPK}^{\prime}$ 's primary role, as a function of the cytoskeleton, is the regulation of actin filament dynamics and organization via control of ADF4 activity.

To further define the role of CPK3 in actin filament organization and immune signaling, we undertook a comprehensive, and quantitative, evaluation of actin filament organization in guard cells from WT Col-0 and the cpk3-2 mutant. As a baseline for PAMP and pathogen-induced reorganization of the actin filament array in stomatal guard cells, uninoculated, naïve WT Col-0 and $c p k 3-2$ mutant plants were first quantitatively evaluated for angular differences in filament orientation. This was achieved by measurements of mean angular difference between actin filament arrays and the stomatal pore edge. Interestingly, we observed that stomatal guard cells have a lower value in the $c p k 3$ 2 mutant plant as compared to WT Col-0 (Fig. 4d), revealing that actin arrays in the cpk3-2 mutant are predominantly longitudinal. In addition, skewness values, which represent the degree of filament bundling, were higher in the cpk3-2 mutant than in WT Col-0 at $0 \mathrm{~h}$ (Fig. $4 \mathrm{~d}-\mathrm{f}$ ). This result is consistent with our observation of filament organization in epidermal pavement cells (Fig. 3a). Additionally, following flg22 and Pst DC3000 inoculation, the angular difference in both WT Col-0 and the cpk3-2 mutant showed a significant reduction in filament angles at $1 \mathrm{hpi}$ (Fig. 4e, f), indicative of the initiation of stomatal closure ${ }^{13}$. After elicitation with $10 \mu \mathrm{M}$ flg22 (Fig. 4f), incremental changes in skewness occurred at 2-to-4 hpi in WT Col-0, suggesting stomatal guard cell filament recovery, a process coincident with guard cell opening. In the cpk3-2 mutant, however, changes in actin filament skewness and occupancy were minor.
Stomatal closure constitutes a functional output of $\mathrm{PTI}^{25}$. Based on previous work ${ }^{13}$, the altered actin filament dynamics and organization in the $c p k 3-2$ guard cell raised the possibility that the cpk3-2 mutant are compromised in stomatal closure response during infection. To define the role of CPK3 in flg22 and Pst DC3000-induced stomatal closure, leaves from WT Col-0 and the cpk3-2 mutant were elicited with flg22 and the stomatal guard cell response was evaluated. Following flg22 and Pst DC3000 elicitation, WT Col-0 stomata rapidly closed (Fig. $4 \mathrm{a}, \mathrm{b}$ ). However, elicitation of $c p k 3-2$ mutant plants with $10 \mu \mathrm{M}$ flg22 did not induce stomatal closure (Fig. 4c); complementation of the cpk3-2 mutant restored the flg22-induced closure response (Fig. 4c and Supplementary Fig. 11). Expression of the kinase-dead variant of CPK3 (i.e., cpk3-2/35S::CPK3 ${ }^{K 107 M}$ ) did not restore flg22-induced stomata closure (Fig. $4 \mathrm{c}$ and Supplementary Fig. 11). These results demonstrate that activation of CPK3 is required for PTI-associated stomata closure in response to flg22 perception.

CPK3 is required for both basal defense against Pst DC3000 and AvrPphB-triggered ETI. Having demonstrated a critical role of CPK3 as a regulator of ADF4-mediated actin filament dynamics and organization during PTI and stomatal closure, we next pursued experiments to gain insight into whether CPK3 contributes to the host resistance against pathogen in terms of or basal defense and ETI. To do this, we first inoculated WT Col-0, cpk3-2, the complementation lines $c p k 3-2 / \mathrm{CPK} 3$ and $c p k 3-2 / \mathrm{CPK} 3{ }^{\mathrm{K} 107 \mathrm{M}}$ with Pst DC3000. As shown, the $c p k 3-2$ mutant and $c p k 3-2 / \mathrm{CPK} 3{ }^{\mathrm{K} 107 \mathrm{M}}$ showed enhanced disease symptom development and significantly 
a
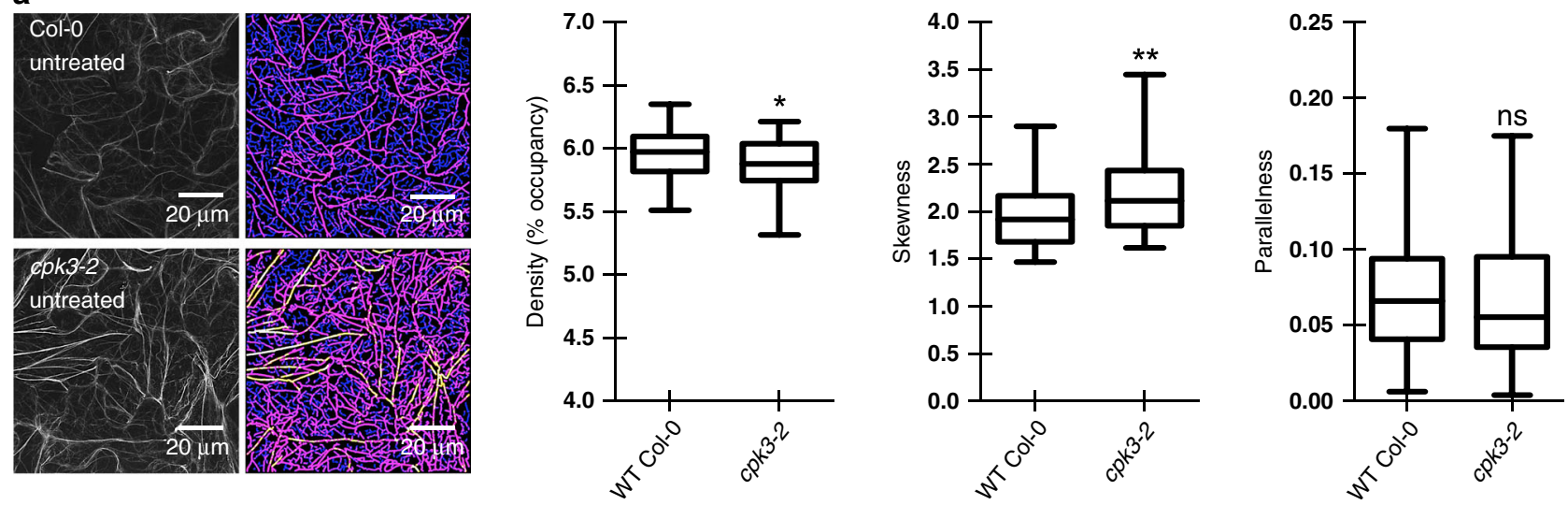

\section{b}


Fig. 3 CPK3 activity modulates ETI- and PTI-induced changes in actin cytoskeletal organization. Quantitative analysis of actin cytoskeletal organization in epidermal pavement cells from a untreated WT Col-0 and the cpk3-2 mutant, mock and Pst DC3000-inoculated b WT Col-0 and the cpk3-2 mutant, and c flg22-elicited WT Col-0 and the cpk3-2 mutant. Representative confocal images (left) and skeletonized images for quantitative evaluation (right) are shown. Images were collected from $\geq 75$ cells. Experiments were performed three times from multiple, independent, biological replicates. The box and whiskers plots represent minimum and maximum values. The line in the box plot represents the median value and the boundaries demonstrate the 25 th percentile (upper) and the 75th percentile (lower). The statistics is performed with two tailed Mann-Whitney $U$-test; ${ }^{*} p$-value $<0.05$; ${ }^{\star *} p$-value $<0.01$; ns, ${ }^{\star \star *} p<0.001 ;{ }^{* \star \star \star} p<0.0001$; ns, not significant.

higher pathogen growth compared to WT Col-0 (Fig. 5a, b and Supplementary Figs. 12 and 13). This, together with additional data (e.g., Supplementary Fig. 7 and Supplementary Fig. 9), indicates that $\mathrm{CPK} 3$, including its kinase activity, is required for the activation of robustness basal defense signaling. We posit that this further illustrates the role of CPK3 in PTI-triggered actin reorganization and stomatal immunity.
To evaluate the role of CPK3 in ETI, and as an extension of our previous work with $\mathrm{ADF} 4^{13,15}$, we next measured the in planta growth of Pst DC3000-AvrPphB. As shown in Fig. 5, we observed a significant increase in susceptibility in $c p k 3-2 / \mathrm{CPK} 3$ and $c p k 3-2 /$ $\mathrm{CPK} 3{ }^{\mathrm{K} 107 \mathrm{M}}$ plant lines (Fig. 5a, b and Supplementary Fig. 13). Next, we evaluated the induction of the ETI-induced HR by infiltrating plants (WT Col-0, cpk3-2, cpk6, and cpk28) with Pst 



Fig. 4 CPK3 activity is required for stomatal immunity and guard cell actin dynamics. Deletion of CPK3 compromises stomatal immunity. Guard cells from the cpk3-2 mutant do not close in response to a flg22 or b Pst DC3000 treatment over a $6 \mathrm{~h}$ time course. Fourteen-day-old seedlings were stimulated with water or $10 \mu \mathrm{M}$ purified flg22 peptide for $1 \mathrm{~h}$, and images were collected by laser scanning confocal microscopy. c CPK3 kinase function is essential for guard cell response to flg22 treatment. Four-week-old WT Col-0, the cpk3-2 mutant, the complementation lines with full kinase function (i.e., cpk3-2/35S:: $\left.C P K 3^{\# 1}\right)$, and the kinase null line (cpk3-2/35S::CPK3 ${ }^{K 107 M \# 1}$ ) were stimulated with $10 \mu \mathrm{M}$ purified flg22 peptide for $1 \mathrm{~h}$. Images were collected by laser scanning confocal microscopy, and stomatal apertures (width/length) were calculated using ImageJ. Error bars represent means \pm SD from three technical replicates of three independent biological repeats $(n \geq 24)$. Statistical analysis was performed using a two tailed Mann-Whitney $U$-test; ${ }^{\star} p<0.05 ;{ }^{\star \star \star} p<$ 0.001; ${ }^{\star \star \star \star} p<0.0001$; ns, not significant). Guard cell actin parameters of WT Col-0/35S::GFP-fABD2 and the cpk3-2/35S::GFP-fABD2 mutant from 14-dayold seedlings inoculated with $\mathbf{d}$ mock, e $3 \times 10^{7} \mathrm{CFU} \mathrm{mL}{ }^{-1}$ Pst DC3000, and $\mathbf{f} 10 \mu \mathrm{M}$ flg22. Left panels are representative images for guard cells. Stomatal images were collected over a $6 \mathrm{~h}$ time interval following inoculation. Images were collected from $\geq 24$ cell pairs, and experiments were performed three times from multiple, independent, biological replicates. The box and whiskers plots represent minimum and maximum values. The line in the box plot represents the median value and the boundaries demonstrate the 25 th percentile (upper) and the 75th percentile (lower). Statistical analysis was conducted using a two tailed Mann-Whitney U-test; ${ }^{*} p$-value $<0.05 ;{ }^{\star \star} p$-value $<0.01$; ns, ${ }^{\star \star \star} p<0.001 ;{ }^{\star \star \star \star} p<0.0001$; ns, not significant.

DC3000, Pst DC3000-AvrPphB, Pst DC3000-AvrRpt2, and Pst DC3000-AvrB (Fig. 5c and Supplementary Fig. 12). Consistent with previous reports showing a blocked $\mathrm{HR}$ in the adf4 mutant ${ }^{13,16}$, we also observed that the HR was abrogated in the cpk3-2 mutant following Pst DC3000-AvrPphB inoculation, yet unaffected in Pst DC3000-AvrRpt2 and Pst DC3000-AvrB inoculated plants (Fig. 5c). Further evaluation of the specificity of CPK3 using the complementation lines revealed a restoration in disease symptom development in $c p k 3-2 / \mathrm{CPK} 3$, but not the kinase inactive-expressing line $c p k 3-2 / \mathrm{CPK} 3^{\mathrm{K} 107 \mathrm{M}}$ (Fig. 5d). 


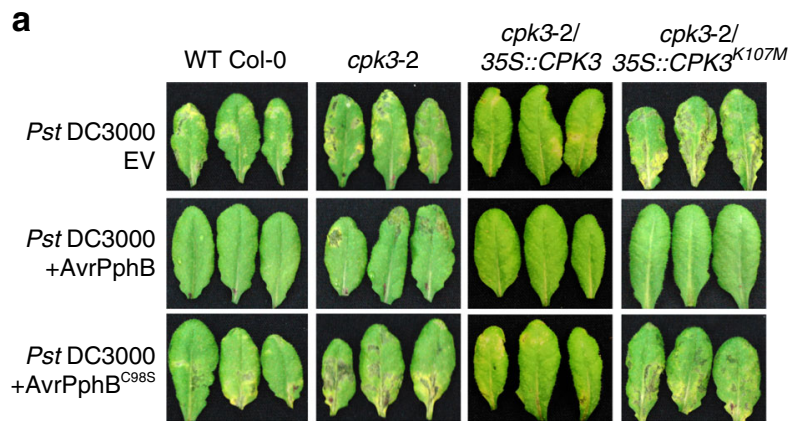

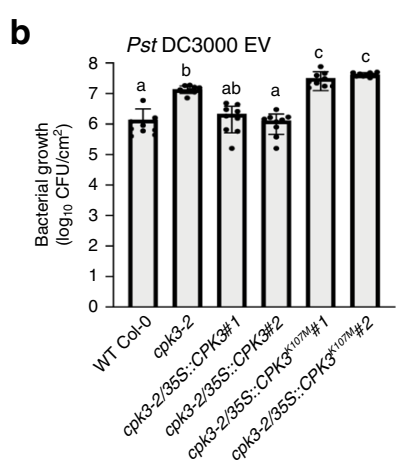

C

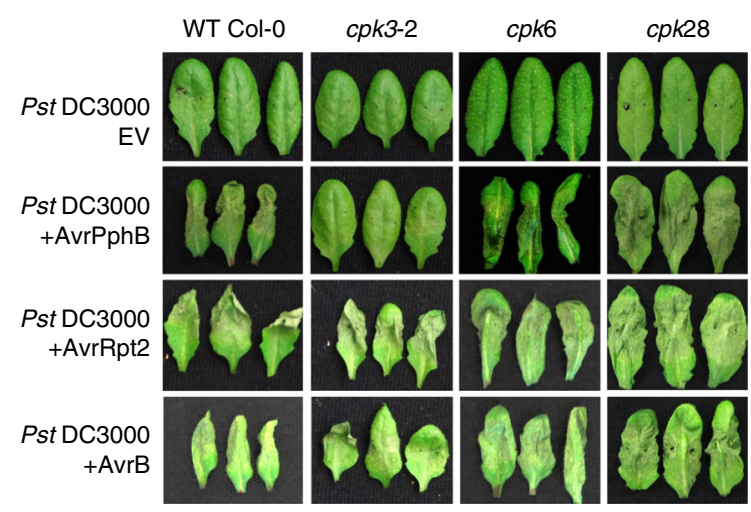







Fig. 5 CPK3 is required for immunity to Pst DC3000-AvrPphB. a CPK3 kinase function is required for disease resistance to Pst DC3000 and Pst DC3000-AvrPphB. Four-week-old plants were dip-inoculated with Pst DC3000 strains $\left[3 \times 10^{7} \mathrm{CFU} \mathrm{mL}^{-1}\right]$. Images were taken at 4 dpi. Three representative leaves are shown from $n \geq 30$. All experiments were performed three times, with similar results. $\mathbf{b}$ In planta bacterial growth was enumerated at 4 days post-inoculation (dpi). Error bars represent means \pm SD from three technical replicates of three independent biological repeats $(n=$ 9, one-way ANOVA; $p<0.05$; ns, not significant). c The CPK3 mutant, cpk3-2, is specifically compromised in the activation of the Pst DC3000-AvrPphBinduced hypersensitive response (HR). Leaves from 4-week-old plants were infiltrated with Pst DC3000-AvrPphB [10 ${ }^{7}$ colony-forming units (CFU mL ${ }^{-1}$ )] using a 1-mL needleless syringe. Images were taken at $24 \mathrm{~h}$ post-inoculation (hpi). CPK6 and CPK28 mutants show WT levels of HR induction following Pst DC3000-AvrPphB inoculation. d CPK3 kinase function is required for HR to Pst DC3000-AvrPphB. Four-week-old plants were infiltrated with Pst



Taken together, these data demonstrate that CPK3 also plays a significant role in AvrPphB-triggered ETI. To investigate whether CPK3 is an upstream signaling component of ADF4-dependent AvrPphB-mediated resistance, we generated a cpk3-2/adf4-2 double mutant and performed bacterial growth curve assays to evaluate ETI. As shown, these data reveal that both $c p k 3-2$ and adf4-2 mutant lines are more susceptible that WT Col-0 against Pst DC3000-AvrPphB, and additionally, that there is not an additive effect (i.e., increased susceptibility) in the $c p k 3-2 / a d f 4-2$ double mutant (Supplementary Fig. 13c). This data supports our hypothesis that $\mathrm{CPK} 3$ and ADF4 likely function within the same immune signaling pathway.

To further define the link between AvrPphB and the activation of resistance through the modulation of host actin cytoskeletal organization, we asked if the function(s) of AvrPphB, RPS5, and/ or PBS1 are associated with an actin-containing complex, in vivo. We did not detect a specific interaction between either PBS1 or RPS5 with actin (Supplementary Fig. 14). However, and quite surprisingly, we identified an interaction between the catalytically inactive AvrPphBC98S and plant actin. This finding suggests another possible link between the HR cell death-inducing activity of the AvrPphB-RPS5 signaling node and a concomitant disruption in actin filament architecture via CPK3-ADF4 (Supplementary Fig. 15a). However, we did not observe a direct impact on actin filament bundling or depolymerization using recombinant AvrPphB protein and purified non-muscle actin, in vitro (Supplementary Fig 15b, c). We posit that these data indicate that during RPS5-mediated ETI, CPK3 may not simply 
function in inhibiting the activity of ADF4 by phosphorylation, but likely involves additional signaling processes, which contribute to the overall depolymerization of actin filaments. In total, these results signify a functional link between AvrPphB, CPK3, and the requirement for actin remodeling during Pst DC3000AvrPphB that would require further investigation.

\section{Discussion}

Previous studies have implicated a role for the plant cytoskeleton during pathogen infection and the activation of immunity ${ }^{10-17,24,26}$. However, key gaps remain in defining a role for cytoskeletal organization during plant defense. In the current study, we undertook an in vivo genetic and cell biology-based approach to define the mechanisms underpinning of the control of actin cytoskeletal organization during pathogen infection. As presented, the data herein provide compelling evidence that ADF4 is a physiological substrate of CPK3, and that phosphorylation of ADF4 by CPK3 is likely a required step in the activation of immune signaling. As a link to the requirement for specific immune-associated changes in cytoskeletal organization, our data also demonstrate that CPK3 is required for pathogen-induced changes in the host actin cytoskeleton. Taken together, the data presented herein support a mechanism for CPK3-mediated control of actin-dependent immune regulation, a process that is targeted by Pst DC3000AvrPphB during infection. Based on this, we propose that CPK3 represents a missing link between actin cytoskeletal organization, PTI, and ETI, and is a key virulence target during pathogen infection. In short, our data support a model whereby changes in actin cytoskeletal organization contribute to the activation of immunity during pathogen infection.

In Arabidopsis, $\sim 70$ actin-binding proteins (ABPs) are required for filament organization ${ }^{9}$. In humans, more than $200 \mathrm{ABPs}$ are required ${ }^{27-29}$. Among these ABPs, members of the actindepolymerizing factor and cofilin family are key regulators of actin cytoskeletal organization ${ }^{25,26}$. ADFs are present in all eukaryotes, with 11 functional ADFs in Arabidopsis, varying in tissue and developmental expression ${ }^{30,31}$. In humans, actin depolymerization is controlled via the activity of 2-cofilins and 1$\mathrm{ADF}^{28}$, and in total, it has demonstrated that depolymerization activity is regulated by a variety of factors, including phosphorylation, the expression and spatial distribution of cofilin, the ratio of globular (G)- versus filamentous (F)-actin, and the relative expression levels and activity of other ABPs. In short, it is hypothesized that actin depolymerization is dependent upon the cellular equilibrium of actin, which includes the spatial and temporal expression of $\mathrm{ABPs}$ and their regulators ${ }^{27}$. As an example of the importance of cofilin/ADF in human disease models, disruptions in the function of this class of protein is associated with numerous diseases, including Alzheimer's, Parkinson's, and DNA damage repair in cancer ${ }^{29,32}$.

In contrast to humans, plants have an expanded number of $\mathrm{ADF}$ genes, which is hypothesized to impart functional specificity based on temporal, tissue, and developmental patterns of expression $^{31}$. Of the $11 \mathrm{ADFs}$ in Arabidopsis, several reports have demonstrated a role for ADF4 in immune signaling in response to bacterial and fungal pathogen infection $13,16,20$. In total, these studies utilized a suite of complementary in vitro biochemical and in vivo microscopic methods for the characterization of ADF4, demonstrating a bona fide, in vivo, actin filament depolymerization function ${ }^{16,33}$. This study, together with previous studies, advances our understanding of the role of ADFs in a variety of immune signaling processes in both plants and animals.

Phosphorylation of ADF and cofilin is indispensable for their functions ${ }^{9,34,35}$. In humans, LIM kinases phosphorylate both cofilin and $\mathrm{ADF}^{36}$. In plants, the identification of the ADF kinase (s) relevant to host-pathogen interactions has remained elusive; however, several candidate kinases have been identified ${ }^{7,8}$. Previous work, together with this study, demonstrates that when unphosphorylated, Arabidopsis ADF4 is able to bind ADP-bound F-actin with high affinity and can sever and depolymerize stabilized filaments ${ }^{13}$. Conversely, phosphorylated ADF4 (and cofilin) exhibits a lower affinity for F-actin filaments, and thus is, therefore, considered inactive. Additional in vivo analyses of two members of the Arabidopsis ADF family supports these in vitro models ${ }^{13,16}$.

Herein, we have identified CPK3 as a kinase responsible for the phosho-regulation of ADF4. Based on the sum of the data presented herein, we proposed that ADF4 is a key executor of pathogen-triggered actin reorganization in plants, and that CPK3 is the regulatory kinase controlling ADF4-mediated actin dynamics during immune signaling. As further support for this mechanism, the finding presented herein extend previous work, which demonstrated that independent disruption of two guard cell-expressed CPK genes-CPK3 and CPK6-lead to impairment of ABA- and $\mathrm{Ca}^{2+}$-dependent stomatal closure ${ }^{37}$, key signaling processes also associated with the activation and regulation of plant immunity. As entry points for bacterial and fungal plant pathogens, stomatal guard cell gating represents a critical point in immune signaling.

Coupled with work previous work by Mori et al. ${ }^{37}$., our data describe a mechanism linking immune-induced changes in actin cytoskeleton organization to the modulation of stomatal closure. Taken together, these data provide evidence linking actin to additional key signaling processes mediated by $\mathrm{CPK}$, including hormone signaling, pathogen virulence, and immunity.

As a mechanism associating CPK3 with ETI, two observations were key in assigning ETI-based functions. First, we identified CPK3 as a target of AvrPphB, as AvrPphB physically interacts with and destabilizes CPK3. The data presented herein support the hypothesis that this is due to either direct enzymatic cleavage of CPK3 by AvrPphB, or potentially, cleavage of unknown CPK3 interactor(s), which in turn lead to destabilization of CPK3. In either case, our data support a mechanism that describes a previously undefined virulence activity for $\mathrm{AvrPphB}$ whereby the reduction of CPK3 results in decreased phosphorylation of ADF4 and less regulatory power to mediate actin remodeling. As a consequence of altered actin cytoskeletal dynamics, immune signaling is abrogated. Interestingly, while we demonstrate that ADF4 phosphorylation inhibits the ADF4-actin interaction, whether such inhibition represents an overall increase in actin filament severing/depolymerization, or F-actin stabilization, remains unanswered.

Taken together, the data presented herein provide a mechanistic understanding of cpk3-2 susceptibility following Pst DC3000AvrPphB infection, one that is underpinned by pathogen-targeting of the CPK3-ADF4 phospho-switch. This is important, as it demonstrates ADF4 phosphorylation is a key step in the transition from a homeostatic surveillance function of the plant actin cytoskeleton to that of an activated immune signaling platform. In support of this hypothesis, we show that $c p k 3-2$ mutant plants inoculated with Pst-AvrPphB show enhanced susceptibility and attenuation of the HR, compared to WT Col- 0 , which phenocopies our previous observation of enhanced susceptibility in the adf4 mutant. Additional $c p k$ mutants tested (e.g., $c p k 6, c p k 10$, and cpk28) were not susceptible to Pst DC3000-AvrPphB. These data provide compelling evidence in support of the hypothesis that PstAvrPphB targets the host immune system through inhibiting CPK3 functionality, a process required for proper function of ADF4 and organization of the actin cytoskeleton. 


\section{Methods}

Plant genotypes and growth. Arabidopsis thaliana plants used in this study includes wild-type (WT) Col-0, the $c p k 3-2$ mutant (SALK-022862 ${ }^{37}$;), the $c p k 6$ mutant (SALK-02546037;), the cpk28 mutant (CS336535 38 ;), the adf4-2 mutant (SALK-121647 ${ }^{8}$;). DNA primers for SALK mutant validation are listed in Supplementary Table 1. Arabidopsis seeds were stratified for $2 \mathrm{~d}$ in the dark at $4^{\circ} \mathrm{C}$ then sown onto soil. All plants were grown in a BioChambers model FLX-37 walkin growth chamber (BioChambers, Manitoba, Canada) at $20^{\circ} \mathrm{C}$ under long day conditions ( $16 \mathrm{~h}$ of light $/ 8 \mathrm{~h}$ of dark) with $60 \%$ relative humidity and a light intensity of $\sim 120 \mu \mathrm{mol}$ photons $\mathrm{m}^{-2} \mathrm{~s}^{-1}$. For mutant complementation, the $c p k 3-2$ mutant was transformed via the floral dip method ${ }^{39}$ with an Agrobacterium tumefaciens strain, GV3101, harboring the binary vector pEarleygate- $203^{40}$, which contained either the full-length open reading frames (ORF) of CPK3 and the CPK3 kinase autophosphorylation mutant variant K107M (i.e., CPK3 ${ }^{\mathrm{K} 107 \mathrm{M}}$ ). Transgenic plants were selected with glufosinate-ammonium (BASTA, MiliporeSigma, Cat \# 45520).

Bacteria growth. Escherichia coli (E. coli) and Agrobacterium tumefaciens clones were grown on Luria-Bertani (LB) medium containing antibiotics as prescribed by plasmid-borne antibiotic resistance markers. Antibiotics were purchased from GoldBio. Pseudomonas syringae pv. tomato DC3000 (Pst DC3000) strains were grown as previously described ${ }^{13}$. Antibiotics (and final concentrations) used in this study include gentamycin (Cat \# G-400-1, $50 \mu \mathrm{g} \mathrm{mL}^{-1}$ ), kanamycin (Cat \# K-120$5,50 \mu \mathrm{g} \mathrm{mL}^{-1}$ ), rifampicin (Cat \# R-120-1, $100 \mu \mathrm{g} \mathrm{mL}^{-1}$ ), spectinomycin (Cat \# S$\left.140-5,50 \mu \mathrm{g} \mathrm{mL}^{-1}\right)$

In planta bacterial growth assays. $P$. syringae strains were either hand-infiltrated using a $1-\mathrm{mL}$ needleless syringe as previously described ${ }^{41}$, or dip-inoculated as described by Kunkel et al. ${ }^{39}$. For in planta bacterial growth enumeration following hand-infiltration of Arabidopsis leaves, Pst DC3000 constructs were inoculated using a $1-\mathrm{mL}$ needleless syringe at a final concentration of $2 \times 10^{5} \mathrm{CFU} \mathrm{mL}^{-1}$. For dip-inoculation-based assays, Pst DC3000 was inoculated at a final concentration of $3 \times 10^{7} \mathrm{CFU} \mathrm{mL} \mathrm{mL}^{-1}$. Samples were collected at 0 - and 72 -h post-inoculation (hpi) for hand-infiltration and $96 \mathrm{hpi}$ for dip-inoculation assays. For analysis of the hypersensitive response (HR), leaves from 4-week-old plants were hand-infiltrated with Pst DC3000 constructs at a concentration of $10^{7} \mathrm{CFU} \mathrm{mL}^{-1}$ using a $1-\mathrm{mL}$ needleless syringe. Leaves were photographed at $20 \mathrm{hpi}$.

For pathogen-associated molecular pattern (PAMP)-triggered immunity (PTI) priming experiments, 4-week-old Arabidopsis plants were hand-infiltrated with flg22 $(1 \mu \mathrm{M})$ and incubated at room temperature (ca. $22^{\circ} \mathrm{C}$ ) for $24 \mathrm{~h}$. After $24 \mathrm{~h}$, flg22-infiltrated leaves were hand-infiltrated with Pst DC3000 strains harboring the vector pVSP61. All pathogen-inoculation experiments were performed at least three times with three technical replicates per experiment. A one-way analysis of variance (ANOVA) using GraphPad Software (Prism) was employed for statistical analysis of in planta bacterial growth in the various plant genotypes.

Cloning and DNA mutagenesis. For protein purification, the open reading frames (ORFs) of $C P K 3, C P K 6, C P K 28, P B S 1, B I K 1$, and $A D F 4$ were amplified by polymerase chain reaction (PCR) from cDNA, which was reverse-transcribed from total RNA isolated from WT Col-0 and cloned into pENTR/D-TOPO (Invitrogen). The resultant ORF constructs were verified by DNA sequencing (MSU Research Technology Support Facility). The sequence confirmed cDNA constructs of interest were cloned into the bacterial expression vector pDEST17 (Invitrogen) using Gateway recombination (i.e., LR Clonase; Invitrogen). Phosphomimetic and phosphor-null derivatives of ADF4, as well as the catalytically inactive CPK3 variant $\mathrm{K} 107 \mathrm{M}$, were created by Gibson cloning according to the manufacturer's instructions (New England Biolabs). All DNA primers used for cloning in this study are listed in Supplementary Table 2.

Protein expression, purification, and kinase assays. Plasmid constructs containing the ORFs of the various CPK and ADF4 derivatives of interest were transformed into OverExpress ${ }^{\text {Th }} \mathrm{C} 41$ (DE3) E. coli competent cells (Lucigen) and the constructs were expressed for $24 \mathrm{~h}$ at $14^{\circ} \mathrm{C}$ (shaking at $225 \mathrm{rpm}$ ). Bacterial cultures were induced with $0.5 \mathrm{mM}$ IPTG (isopropyl $\beta$-D-1-thiogalactopyranoside; MiliporeSigma, Cat \# I6758) at a bacterial culture density of $\mathrm{OD}_{600 \mathrm{~nm}}=0.8$ (ca. $6.4 \times 10^{8} \mathrm{CFU} \mathrm{mL}^{-1}$ ). Proteins were batch purified using Ni-NTA resin (Qiagen) according to the manufacturer's recommendations. Eluted proteins were further purified by fast protein liquid chromatography (FPLC) using a Superdex 200 10/ $300 \mathrm{GL}$ column (GE Healthcare) under physiological conditions $\left(0.05 \mathrm{M} \mathrm{NaH}_{2} \mathrm{PO}_{4}\right.$ and $0.15 \mathrm{M} \mathrm{NaCl}, \mathrm{pH} 7.5)$.

Protein phosphorylation assays reactions consisted of $5 \mu \mathrm{g}$ of substrate, $0.5 \mu \mathrm{g}$ of kinase, $0.5 \mu \mathrm{L}$ of radiolabelled ATP $\left[\gamma^{3}{ }^{32} \mathrm{P}\right]$ (Perkin Elmer), and $4 \mu \mathrm{L}$ of $5 \mathrm{x}$ buffer (0.1 M Tris-HCl, pH 7.5, $50 \mathrm{mM} \mathrm{MgCl}, 1 \mathrm{mM} \mathrm{CaCl}_{2}, 5 \mathrm{mM}$ DTT, 1 complete, mini, EDTA-free protease inhibitor cocktail tablet (MiliporeSigma, Cat \# 11836170001 ; 1 tablet/50 mL homogenization buffer) in a total final volume of $20 \mu \mathrm{L}$. Reactions were incubated for $1 \mathrm{~h}$ at $30^{\circ} \mathrm{C}$ and stopped by the addition of $5 \mathrm{x}$ Laemmli sample buffer. Kinase reactions were resolved by $4-12 \%$ sodium dodecyl sulphate-polyacrylamide gel electrophoresis ((SDS-PAGE) (NuPAGE, Invitrogen)), and gels were stained with $\mathrm{CBB}$ for $1 \mathrm{~h}$ at room temperature $\left(\mathrm{ca} .22^{\circ} \mathrm{C}\right)$, followed by destaining in clearing buffer
(10\% acetic acid, $20 \%$ methanol). Destained gels were dried under a vacuum (Slab Gel Dryer SE1160, Hoefer). Reactions were visualized by detection on X-ray film. Negative control reactions were run in parallel and consisted of each of the aforementioned components yet contained either no kinase or no substrate.

RNA isolation and quantitative real-time PCR. For mRNA expression analyses, 4-week-old Arabidopsis leaves were syringe-infiltrated with flg22 $(1 \mu \mathrm{M})$ or Pst DC3000 strains $\left[3 \times 10^{7} \mathrm{CFU} \mathrm{mL}^{-1}\right]$ using a $1-\mathrm{mL}$ needleless syringe and incubated at $22{ }^{\circ} \mathrm{C}$. For analysis of mRNA accumulation following flg 22 treatment, samples were collected at $0,30 \mathrm{~min}, 1 \mathrm{~h}$, and $3 \mathrm{~h}$ post-inoculation. For Pst DC3000 infection, leaf samples were collected at 0,6 , and $12 \mathrm{~h}$ after inoculation. Total RNA was extracted from samples using the RNeasy Plant Mini kit (Qiagen). One microgram of total RNA was used for first-strand cDNA synthesis using the Maxima H Minus First-Strand cDNA synthesis kit (ThermoFisher Scientific). All DNA primers used for quantitative real-time PCR (qPCR) are listed in Supplementary Table 3. Hot Start SYBR Master Mix 2x (USB Affymetrix) was used to perform qRT-PCR, using an Applied Biosystems 7500 Fast Real-Time PCR System (ThermoFisher Scientific). Amplification of $P P 2 A$ was used as an internal control. Fold expression was calculated as previously described ${ }^{13}$. All mRNA expression data was analyzed using GraphPad Software (Prism). Expression values are represented as mean \pm standard error of the mean (SEM). Statistical analysis was evaluated using a two-way ANOVA, followed by the Bonferroni post-test as compared to WT Col- $0 . p$ values $\leq 0.05$ were considered significant, where ${ }^{*} p$-value $<0.05,{ }^{*} p$-value $<0.01$, and $* * * p$-value $<0.005$.

MAPK phosphorylation assays. For MAPK phosphorylation assays, 14-day-old Arabidopsis seedlings were stimulated with flg22 $(100 \mathrm{nM})$ by floating a solution of flg22 peptide (dissolved in water) onto sterile 14 -day-old seedlings grown on $1 / 2$ MS medium ( $0.7 \%$ agar plates). Seedlings were elicited with flg 22 for $0,10,20,30$, and $60 \mathrm{~min}$ at room temperature (ca. $22^{\circ} \mathrm{C}$ ). At each time point, 15 seedlings were harvested, flash-frozen in liquid nitrogen, and stored at $-80^{\circ} \mathrm{C}$. Samples were processed as previously described ${ }^{13,41}$. Samples $(30 \mu$ g total protein) were resolved by SDS-PAGE and western blot analysis of phosho-MPK3/6 was performed using an anti-pTEpY antibody (Cell Signaling Technology, Cat \# 9101S).

ROS assay. PAMP-induced reactive oxygen species (ROS burst) was measured following a previously reported method ${ }^{42}$, with slight modification. Briefly, leaf disks from 4-week-old Arabidopsis plants were harvested using a 4-mm biopsy punch (Miltex) and floated onto $100 \mu \mathrm{L}$ of sterile $\mathrm{dH}_{2} \mathrm{O}$ in a 96 well-plate, overnight, without motion. After the overnight incubation, the water was carefully removed using a multichannel pipette, and $200 \mu \mathrm{L}$ of a working solution containing $100 \mathrm{nM}$ flg22, $10 \mu \mathrm{g} \mathrm{mL}^{-1} \mathrm{HRP}$ (horse radish peroxidase, MiliporeSigma, Cat \# P8375), and $30 \mu \mathrm{g} \mathrm{mL}^{-1}$ luminol (MiliporeSigma, Cat \# A8511) was added to each well. After addition of the working solution, the plate was immediately moved to a microplate reader (SpectraMax-L, Molecular Devices) for measurement of luminescence. Measurements were taken over a $1 \mathrm{~h}$ time course, with a step of $2 \mathrm{~min}$. Three biological repeats were performed, each with similar results. For each assay, four leaf disks from four individual plants were used. All reaction steps were conducted at room temperature.

Mass spectroscopy analysis. To identify phosphorylated residues in ADF4, $20 \mu \mathrm{g}$ of purified proteins was incubated, individually, with $2 \mu \mathrm{g}$ of purified CPK3, BIK1, and PBS1 in kinase buffer for $1 \mathrm{~h}$. Phosphorylation reactions were separated by SDS-PAGE (15\% Tris-Bis), and resolved gels were stained with CBB. The stained protein bands corresponding to ADF4 or PBS1 ${ }^{\mathrm{K} 115 \mathrm{~N}}$ were excised and was subjected to in-gel digestion according to the method of Shevchenko et al. ${ }^{43}$, with slight modification. Briefly, excised gel bands were dehydrated in $100 \%$ acetonitrile $(\mathrm{ACN})$ and incubated with $10 \mathrm{mM}$ DTT in $100 \mathrm{mM}$ ammonium bicarbonate $(\mathrm{pH}$ 8 ), at $56^{\circ} \mathrm{C}$ for $45 \mathrm{~min}$. After $45 \mathrm{~min}$, the samples were dehydrated again and incubated in the dark with $50 \mathrm{mM}$ iodoacetamide in $100 \mathrm{mM}$ ammonium bicarbonate for $20 \mathrm{~min}$. Gel bands were then washed with ammonium bicarbonate and dehydrated again. Sequencing grade modified trypsin was prepared to $0.01 \mu \mathrm{gLL}^{-1}$ in $50 \mathrm{mM}$ ammonium bicarbonate and $50 \mu \mathrm{L}$ of trypsin was added to each sample such that the gel was completely submerged. Samples were incubated at overnight at $37^{\circ} \mathrm{C}$. Peptides were extracted from the gel by water bath sonication in a solution of $60 \%$ ACN $/ 1 \%$ TCA and vacuum dried to $\sim 2 \mu \mathrm{L}$. Peptide samples were then resuspended in $2 \%$ ACN/0.1\%TFA to $20 \mu \mathrm{L}$. From this, $5 \mu \mathrm{L}$ was injected onto a Thermo Acclaim PepMap RSLC $0.075 \mathrm{~mm} \times 20 \mathrm{~mm}$ C18 trapping column and washed for $\sim 5$ min using a Thermo EASYnLC. Peptides were then eluted onto a Thermo Acclaim PepMap RSLC $0.075 \mathrm{~mm} \times 500 \mathrm{~mm} \mathrm{C18}$ analytical column over $35 \mathrm{~min}$ with a gradient of $2 \%$ B to $40 \%$ B over $24 \mathrm{~min}$, ramping to $100 \%$ B at $25 \mathrm{~min}$ and held at $100 \% \mathrm{~B}$ for the duration of the run (Buffer $\mathrm{A}=99.9 \%$ water $/ 0.1 \%$ formic acid, Buffer $\mathrm{B}=80 \% \mathrm{ACN} / 0.1 \%$ formic acid $/ 19.9 \%$ water).

Eluted peptides were sprayed into a ThermoFisher Q-Exactive HF-X mass spectrometer using a FlexSpray spray ion source. Survey scans were taken in the Orbitrap $(45,000$ resolution, determined at $\mathrm{m} / z$ 200) and the top twenty ions in each survey scan were then subjected to automatic higher energy collision induced dissociation (HCD) with fragment spectra acquired at 7500x resolution. The resultant MS/MS spectra were converted to peak lists using Mascot Distiller (ver 
2.7.0; www.matrixscience.com) and the output was searched against all entries in the TAIR (ver. 10) protein sequence database (downloaded from The Arabidopsis Information Network; www.arabidopsis.org). All entries in the UniProt E. coli protein sequence database (downloaded from www.uniprot.org, 2017-11-01), appended with common laboratory contaminants (downloaded from www.thegpm. org, cRAP project), were scanned using the Mascot searching algorithm (ver. 2.6). The Mascot output was analyzed using Scaffold Q + S (ver. 4.8.4; www. proteomesoftware.com) to probabilistically validate protein identifications. Assignments validated using the Scaffold 1\% FDR confidence filter were considered true.

\section{Construction of gateway vectors for Agrobacterium and protoplast trans-} formation. Construction of the Agrobacterium subcellular localization gateway vectors pBGWB was as follows: pSAT5(A)-DEST-c(175-end)-EYFP-N1 (pE3132) was purchased from TAIR (Cat \#CD3-1096). To restore the XbaI cleavage site from DAM methylation, the sequence between, and including, $X b a \mathrm{I}$ and EcoRI restriction sites was amplified by PCR. After PCR amplification, the PCR products were resolved by gel electrophoresis, digested with $\mathrm{XbaI}$ and $E c o$ RI, and ligated into pNeo1096.

Construction of the protoplast gateway vectors $\mathrm{p} 5 \mathrm{GWH}$ was performed as follows: an ApaI-HA-XbaI and an ApaI-Myc-XbaI dsDNA oligo with sticky ends was generated by annealing two pairs of corresponding DNA primers and ligated into pNeo1096 to generate p5GWH (for HA tag) in place of the original cEYFP. p5GWH::ADF4 was constructed by LR cloning. All DNA primers are listed in Supplementary Table 4.

\section{Agrobacterium-mediated transient expression and co-immunoprecipitation} assays. Sequence confirmed binary vector constructs harboring the ORF of ADF4 (including various derivative mutants) were electroporated into A. tumefaciens strains GV3101 and maintained on LB plates containing rifampicin $\left(50 \mu \mathrm{g} \mathrm{mL}^{-1}\right)$, kanamycin $\left(100 \mu \mathrm{g} \mathrm{mL}^{-1}\right)$, gentamycin $\left(50 \mu \mathrm{g} \mathrm{mL}^{-1}\right)$. For expression of all $A D F 4$ constructs, the cDNAs were cloned into the binary vector pEarleygate- $202^{40}$.

For transient expression in Nicotiana benthamiana, the method of Shimono et al. ${ }^{15}$ was used, with slight modifications. A. tumefaciens GV3101 strains harboring expression constructs of interest were pre-incubated at room temperature (ca. $22^{\circ} \mathrm{C}$ ) in induction media (10 mM MES [pH 5.6], $10 \mathrm{mM} \mathrm{MgCl}_{2}$, $150 \mathrm{mM}$ acetosyringone [MiliporeSigma, Cat \# D134406]) for $2 \mathrm{~h}$ before handinfiltration into 5-week-old $N$. benthamiana leaves using a 1-mL needleless syringe. Inoculated plants were kept at $22^{\circ} \mathrm{C}$ for $40 \mathrm{~h}$ ( $12 \mathrm{~h}$ of light/12 h of dark), after which time 18 leaf discs $\left(1 \mathrm{~cm}^{2} / \mathrm{each}\right)$ were harvested and flash-frozen into liquid nitrogen. For co-immunprecipitation analyses, leaf disks were processed as previously described ${ }^{41}$, with slight modifications. Total protein extracts were isolated by grinding leaf discs in liquid nitrogen into a fine powder, and the powder was transferred to a chilled $\left(4^{\circ} \mathrm{C}\right)$ mortar and further homogenized in homogenization buffer (50 mM HEPES [pH 7.5], $50 \mathrm{mM} \mathrm{NaCl}, 10 \mathrm{mM}$ ethylenediaminetetraacetic acid [EDTA], $0.2 \%$ triton X-100, and 1 cOmplete, mini, EDTA-free protease inhibitor cocktail tablet $[1$ tablet $/ 50 \mathrm{~mL}$ homogenization buffer]). Homogenized samples were centrifuged at $4{ }^{\circ} \mathrm{C}$ at $15,000 \times g$ for $15 \mathrm{~min}$, and $1 \mathrm{~mL}$ of the resultant supernatant was transferred to a sterile 2-mL microcentrifuge tube. Samples were tumbled end-over-end at $4{ }^{\circ} \mathrm{C}$ for $1.5 \mathrm{~h}$ with either $5 \mu \mathrm{L}$ of anti-Flag M2 monoclonal antibody (MilliporeSigma, cat \# F3165) or $10 \mathrm{uL}$ of anti-actin monoclonal antibody (MilliporeSigma, cat \# A3853). After $1.5 \mathrm{~h}$, $50 \mu \mathrm{L}$ of protein-G sepherose-4 fast flow (GE Healthcare), pre-washed [50 mM HEPES (pH 7.5), $50 \mathrm{mM} \mathrm{NaCl}, 10 \mathrm{mM}$ EDTA, $0.1 \%$ triton X-100, and 1 cOmplete mini EDTA-free protease inhibitor cocktail tablet] (was added to each tube and tumbled end-over-end for $4 \mathrm{~h}$ at $4{ }^{\circ} \mathrm{C}$. Input samples were incubated in parallel; these samples did not receive antibodies or protein-G sepharose. After $4 \mathrm{~h}$, samples were centrifuged at $15,000 \times g$ for $15 \mathrm{~s}$, the supernatant discarded, and the pellets were washed 3 times with wash buffer [50 mM HEPES ( $\mathrm{pH} 7.5), 50 \mathrm{mM} \mathrm{NaCl}, 10$ $\mathrm{mM}$ EDTA, $0.1 \%$ triton X-100]. For SDS-PAGE (10\% Bis-Tris) analysis, $25 \mu \mathrm{L}$ of the input sample, $10 \mu \mathrm{L}$ of the immunoprecipitation sample, and $20 \mu \mathrm{L}$ of the coimmunprecipitation samples were separated by electrophoresis, and resolved samples were transferred to nitrocellulose (BioRad, Cat \# 1620115). Signals were detected using the Super Signal West Pico Chemiluminescent Substrate (ThermoFisher, Cat \# 34577), with anti-Flag M2-peroxidase (MilliporeSigma, Cat \# A8592).

ADF4-actin co-localization. $A D F 4$ (including various derivative mutants) and fABD2 were cloned in to $p G W B 555^{44}$ and $p A R T 27^{10}$, respectively, and the Agrobacterium-mediated transient expression in $N$. benthamiana was conducted following the procedure stated above. Leaf disks from $48 \mathrm{hpi} \mathrm{samples} \mathrm{were} \mathrm{observed}$ and photographed using a 60x/1.42 PlanApo N objective and a 100x/1.40 objective on an Olympus FV1000D.

Mesophyll cell protoplast preparation and transformation. For protoplast preparation, 5-week-old Arabidopsis leaves were harvested following previously described methods ${ }^{45}$. Approximately $10^{5}$ protoplasts were transformed with $10 \mu \mathrm{g}$ of plasmid DNA containing various expression constructs of interest. Following transformation, protoplasts were incubated at room temperature under weak light for $14 \mathrm{~h}$, after which time samples were harvested and processed for analysis.

Two-dimensional gel electrophoresis. Five-week-old Arabidopsis leaves were harvested from both WT Col-0 and the $c p k 3-2$ mutant for protoplast preparation as described above. For 2DGE, $10^{5}$ protoplasts were transformed with $10 \mu \mathrm{g}$ of p5GWH::ADF4, and after $14 \mathrm{~h}$ incubation at room temperature. $200 \mu \mathrm{g}$ of HAADF4 protein was isolated for both WT Col-0 and cpk3-2 mutant transformed protoplasts. Total protein extracts were precipitated with chloroform:methanol (1:4) and reconstituted in urea buffer [7 M urea, $2 \mathrm{M}$ thiourea, 2\% CHAPS, 2\% ASA-14, $50 \mathrm{mM}$ DTT, 0.2\% Biolyte ampholytes and 0.1\% bromophenol blue). Isoelectric focusing and SDS-PAGE were conducted according to the manufacturer's instructions (BioRad). Immunoblot analysis was performed described above. The detection of HA-ADF4 was performed by western blot analysis using anti-HA-HRP antibodies (MilliporeSigma, Cat \# 12013819001).

Stomatal closure assays. Four-week-old Arabidopsis plants were used for stomatal closure assays. In brief, leaves were floated in $\mathrm{MES} / \mathrm{KOH}$ buffer $[50 \mathrm{mM} \mathrm{KCl}$ $10 \mu \mathrm{M} \mathrm{CaCl}_{2}, 0.01 \%$ Tween $20,10 \mathrm{mM}$ MES (pH 6.15)] and incubated at $22^{\circ} \mathrm{C}$ in a Percival incubator with constant light for $3 \mathrm{~h}$. Once stomatal apertures stabilized, leaves were incubated under the same conditions, with mock or $10 \mu \mathrm{M}$ flg22 for 1 h. For time course analysis of stomata closure, 10-to-13-day-old Arabidopsis seedlings were used. Plants were treated with $1 \mu \mathrm{M}$ flg22 or Pst DC3000 by dipinoculation. Images of lower epidermal leaf surfaces were observed, and images were collected using an Olympus FV1000D fluorescent confocal microscope (405 $\mathrm{nm}$ laser to measure autofluorescence of the cell walls from the guard cell, and a $488 \mathrm{~nm}$ laser to measure the GFP-fABD2 signal in transgenic plants). The ratio of stomatal aperture (width/length) was analyzed using ImageJ.

G-actin/F-actin in vivo assay and actin disruption assay. To monitor the ratio of G-actin versus F-actin in WT Col- 0 and the cpk3 mutant after the activation of immune signaling, a commercially available quantification kit was used, and Gactin/F-actin in vivo assays were performed according the manufacturer's instructions with slight modification. Isolate of G-actin and F-actin from Arabidopsis leaves was performed using G-actin/F-actin in vivo assay kit (Cytoskeleton, Inc., Cat \# BK037). G-actin and F-actin fractions were separated alongside 500, 200, and 75 ng of G-actin standards (bovine cardiac muscle actin, Cytoskeleton, Inc., cat \# AD99-A) on an SDS polyacrylamide gel (4-12\% Bis-Tris gel, Invitrogen, Carlsbad, CA). Relative actin quantification was determined by western blot analysis using monoclonal anti-actin (plant) antibody (MiliporeSigma, CAT \# A0480$25 \mu \mathrm{L})$.

To monitor F-actin disruption, AvrPphB-6xHis and AvrPphBC98S-6xHis were expressed in E. coli $(\mathrm{C} 41, \mathrm{DE} 3)$ and purified as described above for CPK3 and ADF4. Actin disruption and polymerization assays were performed according to the manufacturer's instructions (Cytoskeleton Inc, Cat \# BK013). G- and F-actin fractions were isolated by ultracentrifugation $(150,000 \times g)$ and proteins were separated by SDS polyacrylamide gel electrophoresis (4-12\% Bis-Tris gel, Invitrogen). Relative actin quantification was determined by CBB staining using know amounts of commercially available protein standards (Cytoskeleton Inc).

Confocal microscopy and quantitative evaluation of actin filament organization. To evaluate actin organization including density, bundling and orientation analyses were performed according to previously described methods ${ }^{46-48}$. All image collection and quantitative analyses were performed as double-blind experiments, in biological triplicate. Actin filament analyses were performed on confocal microscopy imaged fields of epidermal pavement cells and guard cells. Images were collected using laser confocal scanning microscopy by obtaining $27 \mathrm{z}-$ series sections at $0.5 \mu \mathrm{m}$ intervals. Laser confocal scanning microscopy was performed using a 60x/1.42 PlanApo N objective on an Olympus FV1000D. Serial optical sections were projected with maximum intensity and analyzed in ImageJ using algorithms previously described ${ }^{46-48}$. Gaussian blur and bandpass filters were applied to stack images before generating projection images for density analysis. Statistical analyses were performed using the Mann-Whitney $U$-test with GraphPad software (Prism). Density, also referred to as "Percent Occupancy", indicates the relative abundance of actin filament signal within a cell. Skewness is an indirect measure of the degree of actin filament bundling. Parallelness indicates variation in actin cytoskeletal orientation ${ }^{47,48}$.

Immunocomplex kinase assays. Protoplasts isolated from $c p k 3-2 / 35 S:: C P K 3$ were digested with $0.5 \mathrm{~mL}$ of immunoprecipitation buffer $(50 \mathrm{mM}$ Tris $\mathrm{HCl}, \mathrm{pH} 7.5,150$ $\mathrm{mM} \mathrm{NaCl}, 5 \mathrm{mM}$ EDTA, $1 \mathrm{mM}$ DTT, $1 \%$ Triton, and 1 cOmplete, mini, EDTAfree protease inhibitor cocktail tablet ( 1 tablet $/ 50 \mathrm{~mL}$ immunoprecipitated buffer) After centrifugation at $15,000 \times g$ for $10 \mathrm{~min}$ at $4{ }^{\circ} \mathrm{C}$, the supernatant was incubated with anti-Myc antibody (AbCam, Cat\# ab32) for $2 \mathrm{~h}$ and then gently tumbled with protein-G-agarose beads for $2 \mathrm{~h}$ at $4{ }^{\circ} \mathrm{C}$. The beads were collected and washed twice with immunoprecipitation buffer and once with kinase buffer $(20 \mathrm{mM}$ Tris. $\mathrm{HCl}, \mathrm{pH} 7.5,10 \mathrm{mM} \mathrm{MgCl}, 200 \mathrm{nM} \mathrm{CaCl}_{2}, 1 \mathrm{mM}$ DTT and cOmplete mini, EDTA-free protease inhibitor). The washed beads incubated in $20 \mu \mathrm{L}$ of kinase buffer with $1 \mu \mathrm{g}$ of myelin basic protein (MBP, MiliporeSigma, Cat \# M1891) and 
$0.5 \mu \mathrm{L}$ of ATP $\left[\gamma^{-}{ }^{32} \mathrm{P}\right]$ at $30^{\circ} \mathrm{C}$ for $1 \mathrm{~h}$ with gentle shaking. Kinase reactions were analyzed by $4-12 \%$ SDS-PAGE (NuPAGE, Invitrogen).

Reporting summary. Further information on research design is available in the Nature Research Reporting Summary linked to this article.

\section{Data availability}

The authors declare that all data supporting the findings of this study are included in the manuscript and its supplementary files or are available from the corresponding author upon request. Source data are provided with this paper.

Received: 22 December 2018; Accepted: 10 November 2020;

Published online: 04 December 2020

\section{References}

1. Blanchoin, L., Boujemaa-Paterski, R., Sykes, C. \& Plastino, J. Actin dynamics, architecture, and mechanics in cell motility. Physiol. Rev. 94, 235-263 (2014).

2. Lee, S. \& Dominguez, R. Regulation of actin cytoskeleton dynamics in cells. Mol. Cells 29, 311-325 (2010).

3. Arber, S. et al. Regulation of actin dynamics through phosphorylation of cofilin by LIM-kinase. Nature 393, 805-809 (1998)

4. Kanellos, G. \& Frame, M. C. Cellular functions of the ADF/cofilin family at a glance. J. Cell Sci. 129, 3211-3218 (2016).

5. Yang, N. et al. Cofilin phosphorylation by LIM-kinase 1 and its role in Racmediated actin reorganization. Nature 393, 809-812 (1998).

6. Allwood, E. G., Smertenko, A. P. \& Hussey, P. J. Phosphorylation of plant actin-depolymerising factor by calmodulin-like domain protein kinase. FEBS Lett. 499, 97-100 (2001).

7. Dong, C. H. \& Hong, Y. Arabidopsis CDPK6 phosphorylates ADF1 at Nterminal serine 6 predominantly. Plant Cell Rep. 32, 1715-1728 (2013).

8. Zhao, S. et al. Casein kinase1-like protein 2 regulates actin filament stability and stomatal closure via phosphorylation of actin depolymerizing factor. Plant Cell 28, 1422-1439 (2016).

9. Day, B., Henty, J. L., Porter, K. J. \& Staiger, C. J. The pathogen-actin connection: a platform for defense signaling in plants. Annu. Rev. Phytopathol. 49, 483-506 (2011).

10. Henty-Ridilla, J. L. et al. The plant actin cytoskeleton responds to signals from microbe-associated molecular patterns. PLoS Pathog. 9, el003290 (2013).

11. Porter, K. \& Day B. Actin branches out to link pathogen perception and host gene regulation. Plant Sign. Behav. 8, e23468 (2013).

12. Porter, K. \& Day, B. From filaments to function: the role of the plant actin cytoskeleton in pathogen perception, signaling, and immunity. J. Int. Plant Biol. 58, 299-311 (2015).

13. Porter, K. J., Shimono, M., Tian, M. \& Day, B. Arabidopsis actindepolymerizing factor-4 links pathogen perception, defense activation and transcription to cytoskeletal dynamics. PLoS Pathog. 8, e1003006 (2012).

14. Shimono, M. et al. Quantitative evaluation of stomatal cytoskeletal patterns during the activation of immune signaling in Arabidopsis thaliana. PLoS ONE 11, e0159291 (2016).

15. Shimono, M. et al. The Pseudomonas syringae type-III effector HopG1 induces actin remodeling to promote symptom development and susceptibility during infection. Plant Physiol. 171, 2239-2255 (2016).

16. Tian, M. et al. Arabidopsis actin-depolymerizing factor AtADF4 mediates defense signal transduction triggered by the Pseudomonas syringae effector AvrPphB. Plant Physiol. 150, 815-824 (2009).

17. Li, P. \& Day, B. Battlefield cytoskeleton: turning the tide on plant immunity. Mol. Plant-Microbe Interact. 32, 25-34 (2019).

18. Shao, F. et al. Cleavage of Arabidopsis PBS1 by a bacterial type III effector. Science 301, 1230-1233 (2003)

19. Warren, R. F., Merritt, P. M., Holub, E. \& Innes, R. W. Identification of three putative signal transduction genes involved in $\mathrm{R}$ gene-specified disease resistance in Arabidopsis. Genetics 152, 410-412 (1999).

20. Zhang, J. et al. Receptor-like cytoplasmic kinases integrate signaling from multiple plant immune receptors and are targeted by a Pseudomonas syringae effector. Cell Host Microbe 7, 290-301 (2010).

21. Veronese, P. et al. The membrane-anchored botrytis-induced kinasel plays distinct roles in Arabidopsis resistance to necrotrophic and biotrophic pathogens. Plant Cell 18, 257-273 (2006).

22. Gomez-Gomez, L. \& Boller, T. FLS2: an LRR receptor-like kinase involved in teh perception of the bacterial elicitor flagellin in Arabidopsis. Mol. Cell 5, 1003-1011 (2000).

23. Zipfel, C. Plant pattern-recognition receptors. Trends Immunol. 35, 345-351 (2014).
24. Kang, Y. et al. HopW1 from Pseudomonas syringae disrupts the actin cytoskeleton to promote virulence in Arabidopsis. PLoS Pathog. 10, e1004232 (2014).

25. Melotto, M., Zhang, L., Oblessuc, P. R. \& He, S. Y. Stomatal defense a decade later. Plant Physiol. 174, 561-571 (2017).

26. Tang, C. et al. TaADF3, an actin depolymerizing factor, negatively modulates wheat resistance against Puccinia striiformis. Front. Plant Sci. 6, 1214 (2015).

27. Andrianantoandro, E. \& Pollard, T. D. Mechanism of actin filament turnover by severing and nucleation at different concentrations of ADF/cofilin. Mol. Cell 24, 13-23 (2006).

28. Bamburg, J. \& Bernstein, B. Roles of ADF/cofilin in actin polymerization and beyond. F1000 Biol. Rep. 2, 1-7 (2010).

29. Gross, S. R. Actin binding proteins: their ups and downs in metastatic life. Cell Adhes. Migrat. 7, 199-213 (2013).

30. Kandasamy, M. K., Burgos-Rivera, B., McKinney, E. C., Ruzicka, D. R. \& Meagher, R. B. Class-specific interaction of profilin and ADF isovariants with actin in the regulation of plant development. Plant Cell 19, 3111-3126 (2007).

31. Ruzicka, D. R., Kandasamy, M. K., McKinney, E. C., Burgos-Rivera, B. \& Meagher, R. B. The ancient subclasses of Arabidopsis actin depolymerizing factor genes exhibit novel and differential expression. Plant J. 52, 460-472 (2007).

32. Chang, C. Y., Leu, J. D. \& Lee, Y. J. The actin depolymerizing factor (ADF)/ cofilin signaling pathway and DNA damage responses in cancer. Int. J. Mol. Sci. 16, 4095-4120 (2015).

33. Henty, J. L. et al. Arabidopsis actin depolymerizing factor4 modulates the stochastic dynamic behavior of actin filaments in the cortical array of epidermal cells. Plant Cell 23, 3711-3726 (2011).

34. Bernard, O. LIM kinases, regulators of actin dynamics. Int. J. Biochem. Cell Biol. 39, 1071-1076 (2007).

35. Lawler, S. Regulation of actin dynamics: the LIM kinase connection. Curr Biol. 9, R800-R802 (1999).

36. Meng, Y. et al. Regulation of ADF/cofilin phosphorylation and synaptic function by LIM-kinase. Neuropharm 47, 746-754 (2004).

37. Mori, I. C. et al. CDPKs CPK6 and CPK 3 function in ABA regulation of guard cell S-type anion- and $\mathrm{Ca}(2+)$-permeable channels and stomatal closure. PLoS Biol. 4, e327 (2006).

38. Monaghan, J. et al. The calcium-dependent protein kinase CPK28 buffers plant immunity and regulates BIK1 turnover. Cell Host Microbe 16, 605-615 (2014).

39. Kunkel, B. N., Bent, A. F., Dahlbeck, D., Innes, R. W. \& Staskawicz, B. J. RPS2, an Arabidopsis disease resistance locus specifying recognition of Pseudomonas syringae strains expressing the avirulence gene avrRpt2. Plant Cell 5, 865-875 (1993).

40. Earley, K. W. et al. Gateway-compatible vectors for plant functional genomics and proteomics. Plant J. 45, 616-629 (2006).

41. Knepper, C., Savory, E. A. \& Day, B. Arabidopsis NDR1 is an integrin-like protein with a role in fluid loss and plasma membrane-cell wall adhesion. Plant Physiol. 156, 286-300 (2011).

42. Sang, Y. \& Macho, A. P. Analysis of PAMP-triggered ROS burst in plant immunity. Methods Mol. Biol. 1578, 143-153 (2017).

43. Shevchenko, A., Wilm, M., Vorm, O. \& Mann, M. Mass spectrometric sequencing of proteins silver-stained polyacrylamide gels. Anal. Chem. 68, 850-858 (1996).

44. Nakagawa, T. et al. Development of series of gateway binary vectors, pGWBs for realizing efficient construction of fusion genes for plant transformation. J. Biosci. Bioeng. 104, 34-41 (2007)

45. Wu, F. H. et al. Tape-Arabidopsis Sandwich-a simpler Arabidopsis protoplast isolation method. Plant Methods 5, 16 (2009).

46. Higaki, T. Quantitative evaluation of cytoskeletal organizations by microscopic image analysis. Plant Morph. 29, 15-21 (2017).

47. Higaki, T., Kutsuna, N., Sano, T., Kondo, N. \& Hasezawa, S. Quantification and cluster analysis of actin cytoskeletal structures in plant cells: role of actin bundling in stomatal movement during diurnal cycles in Arabidopsis guard cells. Plant J. 61, 156-165 (2010).

48. Lu, Y. J. \& Day, B. Quantitative evaluation of plant actin cytoskeletal organization during immune signaling. Methods Mol. Biol. 1578, 207-221 (2017).

\section{Acknowledgements}

This research was supported by a grant (IOS-1557437) from Plant Biotic Interactions, a joint program of the U.S. National Science Foundation (NSF) and USDA's National Institute of Food and Agriculture (NIFA) to B.D. and S.Y.H., and the National Institutes of General Medical Sciences (1R01GM125743) to B.D. We thank Doug Whitten (MSU Research Technology Support Facility Mass Spectroscopy Laboratory) and Rikako Yamauchi (MSU and Meiji University) for technical assistance, and all members of the Day laboratory for discussions and comments during completion of this study. 


\section{Author contributions}

Y.-J.L. and B.D. designed and conceived the experiments. Y.-J.L., P.L., M.S., A.C., T.H., and B.D. performed experiments. Y.-J.L., P.L., M.S., A.C., T.H., S.Y.H., and B.D. analyzed the data. Y.-J.L., S.Y.H., and B.D. wrote the manuscript. All authors commented and agreed on the manuscript before submission.

\section{Competing interests}

The authors declare no competing interests.

\section{Additional information}

Supplementary information is available for this paper at https://doi.org/10.1038/s41467020-20007-4

Correspondence and requests for materials should be addressed to B.D.

Peer review information Nature Communications thanks the anonymous reviewers for their contribution to the peer review of this work.
Reprints and permission information is available at http://www.nature.com/reprints

Publisher's note Springer Nature remains neutral with regard to jurisdictional claims in published maps and institutional affiliations.

\section{(c) (i)}

Open Access This article is licensed under a Creative Commons Attribution 4.0 International License, which permits use, sharing, adaptation, distribution and reproduction in any medium or format, as long as you give appropriate credit to the original author(s) and the source, provide a link to the Creative Commons license, and indicate if changes were made. The images or other third party material in this article are included in the article's Creative Commons license, unless indicated otherwise in a credit line to the material. If material is not included in the article's Creative Commons license and your intended use is not permitted by statutory regulation or exceeds the permitted use, you will need to obtain permission directly from the copyright holder. To view a copy of this license, visit http://creativecommons.org/licenses/by/4.0/.

(C) The Author(s) 2020 